\title{
Article
}

\section{Some New Results on Bicomplex Bernstein Polynomials}

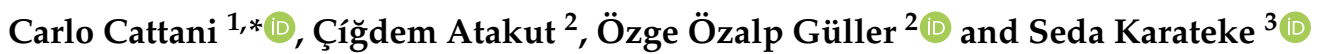 \\ 1 Engineering School, DEIM, Tuscia University, 01100 Viterbo, Italy \\ 2 Department of Mathematics, Ankara University, Ankara 06100, Turkey; atakut@science.ankara.edu.tr (Ç.A.); \\ ozgeguller2604@gmail.com (Ö.Ö.G.) \\ 3 Department of Computer Engineering, Istanbul Ayvansaray University, Istanbul 34087, Turkey; \\ sedakarateke@ayvansaray.edu.tr \\ * Correspondence: cattani@unitus.it
}

Citation: Cattani, C.; Atakut, Ç.; Özalp Güller, Ö.; Karateke, S. Some New Results on Bicomplex Bernstein Polynomials. Mathematics 2021, 9, 2748. https://doi.org/10.3390/math 9212748

Academic Editor: Clemente Cesarano

Received: 22 September 2021

Accepted: 25 October 2021

Published: 29 October 2021

Publisher's Note: MDPI stays neutral with regard to jurisdictional claims in published maps and institutional affiliations.

\begin{abstract}
The aim of this work is to consider bicomplex Bernstein polynomials attached to analytic functions on a compact $\mathbb{C}_{2}$-disk and to present some approximation properties extending known approximation results for the complex Bernstein polynomials. Furthermore, we obtain and present quantitative estimate inequalities and the Voronovskaja-type result for analytic functions by bicomplex Bernstein polynomials.
\end{abstract}

Keywords: bicomplex Bernstein polynomials; analytic functions; holomorphic functions; Voronovskajatype theorem

MSC: 30G35; 41A10; 41A25; 41A36

\section{Introduction}

The approximation theory might be considered as a numerical approach to mathematical analysis problems with wide theoretical methods (see [1,2]). The basic idea behind the approximation theory is to obtain a representation of any arbitrary function in terms of simple fundamental functions (usually polynomials) with the known properties. One of the most fundamental polynomials, the celebrated one of this theory, is the so-called Bernstein polynomials, which can be used to uniformly approximate any continuous function $f \in C[0,1]$ defined on the interval $[0,1]$ (see [3]). Moreover, the first asymptotic formulation of the pointwise approximation of continuous functions whose second derivatives exist at a point $x$ on $[0,1]$ was presented by Voronovskaja (see [4]). Determination of the convergence rate of the sequences of linear positive operators converging to the function $f$ is a key point in the approximation theory. Thus, the following limit, which is known as Voronovskaja formula:

$$
\lim _{m \rightarrow \infty} m\left(L_{m}(f ; x)-f(x)\right),
$$

helps us get information about the convergence rate, where $\left(L_{m}\right)_{m \geq 1}$ is a sequence of linear positive operators. Until today, the generalized cases of Voronovskaja theorems have been widely studied for many operators, which represent various versions of classical Bernstein operators. All of these theorems, are called Voronovskaja-type theorems (see [5-7]). Here, motivated from the Voronovskaja formula, our purpose is to obtain a Voronovskaja-type result in the space of bicomplex numbers using bicomplex Bernstein operators mentioned in Section 3.

Let us start with the complex Bernstein polynomials for $f$ of order $n$, which are defined as follows (see [8]): if $f:[0,1] \rightarrow \mathbb{C}$ is a function, $n$ is a positive integer, $k=0,1,2, \ldots, n$, and then for $z \in \mathbb{C}$ :

$$
B_{n}(f)(z)=\sum_{k=0}^{n}\left(\begin{array}{l}
n \\
k
\end{array}\right) z^{k}(1-z)^{n-k} f\left(\frac{k}{n}\right), \quad z \in \mathbb{C}
$$


where $\left(\begin{array}{l}n \\ k\end{array}\right)$ denotes $\frac{n !}{k !(n-k) !}$.

The approximation properties of complex Bernstein polynomials were primarily studied by Lorentz [3]. Recently, Gal [9] contributed to the estimates for the convergence results of Equation (1) to an analytic function $f$ in closed disks in $[9,10]$. Other Bernsteintype operators of complex variables have been widely studied in [11].

Although the growing interest for such operators (see, e.g., [11] and the references therein), bicomplex Bernstein-type operators have not yet been deeply investigated.

Some approximation results for the bicomplex beta operators for holomorphic functions in strips of a compact disk were given in [12]. However, in general, the literature containing the approximation results of bicomplex versions of these kinds of operators is still very limited. At this point, we want to bridge a gap in the literature by using our study as a benchmark. Nevertheless, there are some recent developments of the bicomplex theory in some applications such as the solution of differential equations, quantum mechanics, biomathematics, Hopefield neural networks and signal theory (see, e.g., $[13,14])$.

The concept of a bicomplex number was given by Segre [15] in 1892, with the aim of describing physical problems in a four-dimensional space. The set of bicomplex numbers is a suitable generalization of complex numbers and hyperbolic numbers, and extensive study may be found in $[16,17]$. Bicomplex algebra is a two-dimensional Clifford algebra also satisfying the commutative rule of multiplication on $\mathbb{C}$ and, it has important applications in numerous fields, such as digital image processing, geometry and theoretical physics (see [17-23]). As a matter of fact, bicomplex theory and bicomplex algebra are developing very rapidly.

The definition of holomorphic functions of a bicomplex variable was given by $\mathrm{Fu}-$ tagawa in 1928 [24,25]. Bicomplex polynomials enjoy some interesting properties. For instance, a bicomplex polynomial of degree $n$ does not necessarily have $n$ zeros. Thus, it can have a finite number of zeros, as well as an infinite number of zeros [26].

In this paper, our main contribution is to give some novel results about the bicomplex Bernstein polynomials in the approximation theory. Some important inequalities fulfilled by bicomplex Bernstein polynomials and a Voronovskaja type-theorem for analytic functions by these polynomials has been proven.

This article is divided into the following sections: Section 2 contains some introductory material on the bicomplex algebra and bicomplex analysis. Section 3 delves into the heart of this study, defining first the bicomplex form of Bernstein polynomials. Then, we provide a quantitative upper estimate for the bicomplex Bernstein operators and their derivatives on the compact $\mathbb{C}_{2}$-disk and establish the qualitative Voronovskaja-type result for these operators. In Section 4, we address some of our recent discussions and upcoming endeavors. Finally, Appendix is divided into three parts labeled Appendices A-C. The proofs of some theorems in Section 3 are included here to facilitate the reader's comprehension.

\section{Preliminaries on Bicomplex Numbers and Bicomplex Polynomials}

In this section, we recall some basic notations $\mathbb{R}, \mathbb{C}$ and $\mathbb{C}_{2}$ for the spaces of real, complex and bicomplex numbers, respectively. As it is well known, an element from the space $\mathbb{C}$ is represented as $x_{1}+i_{1} x_{2}$, such that $x_{1}, x_{2} \in \mathbb{R}, i_{1}{ }^{2}=-1$. On the other hand, an element from the space $\mathbb{C}_{2}$ is written by the basis $1, i_{1}, i_{2}, i_{1} i_{2}$ as follows:

$$
x_{1}+i_{1} x_{2}+i_{2} x_{3}+i_{1} i_{2} x_{4}, \quad x_{1}, \ldots, x_{4} \in \mathbb{R},
$$

where $i_{1}, i_{2}$ and $i_{1} i_{2}$ satisfy the properties $i_{1}{ }^{2}=-1, i_{2}{ }^{2}=-1, i_{1} i_{2}=i_{2} i_{1}$ with $\left(i_{1} i_{2}\right)^{2}=1$. Note that we can see that $i_{1} i_{2} \in \mathbb{C}_{2}$, but $i_{1} i_{2} \notin \mathbb{C}$. From Equation (2), we can also see that since $\mathbb{C}$ is of dimension two over $\mathbb{R}$, the space of bicomplex numbers $\mathbb{C}_{2}$ is an algebra over $\mathbb{R}$ with dimension four.

Let $z_{1}=x_{1}+i_{1} x_{2}$ and $z_{2}=x_{3}+i_{1} x_{4}$ be complex numbers in $\mathbb{C}$. In this case, the element in Equation (2) can be represented as $z_{1}+i_{2} z_{2}$, where $z_{1}, z_{2} \in \mathbb{C}, i_{2}{ }^{2}=-1$. In 
addition, an element which is equal to its square is called as idempotent element. There are four idempotent elements in $\mathbb{C}_{2}$; they are:

$$
0,1, e_{1}=\frac{1+i_{1} i_{2}}{2}, e_{2}=\frac{1-i_{1} i_{2}}{2} .
$$

The idempotent elements $e_{1}, e_{2}$ in Equation (3) have the following properties:

(i) $e_{1} e_{2}=0$,

(ii) $\left\|e_{1}\right\|=\left\|e_{2}\right\|=\frac{\sqrt{2}}{2}$,

(iii) $\left(e_{1}\right)^{2}=e_{1},\left(e_{2}\right)^{2}=e_{2}$,

and every element $\zeta:\left(z_{1}+i_{2} z_{2}\right)$ in $\mathbb{C}_{2}$ has the idempotent representation:

$$
\zeta=\left(z_{1}-i_{1} z_{2}\right) e_{1}+\left(z_{1}+i_{1} z_{2}\right) e_{2}
$$

(see [17]), i.e., for every $\zeta:\left(z_{1}+i_{2} z_{2}\right) \in \mathbb{C}_{2}$,

$$
z_{1}+i_{2} z_{2}=\left(z_{1}-i_{1} z_{2}\right) e_{1}+\left(z_{1}+i_{1} z_{2}\right) e_{2} .
$$

Furthermore, if $\|\cdot\|$ denotes the norm of elements in $\mathbb{C}_{2}$, then:

$$
\left\|z_{1}+i_{2} z_{2}\right\|=\left(\frac{\left|z_{1}-i_{1} z_{2}\right|^{2}+\left|z_{1}+i_{1} z_{2}\right|^{2}}{2}\right)^{\frac{1}{2}} .
$$

There is an important difference between $\mathbb{C}$ and $\mathbb{C}_{2}$ : the complex numbers form a field, but the bicomplex numbers do not since they contain divisors of zero.

So far we have given a basic knowledge of bicomplex numbers and a brief overview of the literature of bicomplex analyses. We should also point the reader to [17,27-29], where fundamental work about the bicomplex numbers, bicomplex analyses and more have been reported.

Let us give the following basic definitions and theorem used throughout this article.

Definition 1 (see [17]). A cartesian set $\mathbb{U}$ in $\mathbb{C}_{2}$ is determined by sets $\mathbb{U}_{1}$ in $A_{1}$ and $\mathbb{U}_{2}$ in $A_{2}$ as follows:

$$
\mathbb{U}=\left\{z_{1}+i_{2} z_{2} \in \mathbb{C}_{2}: z_{1}+i_{2} z_{2}=\left(z_{1}-i_{1} z_{2}\right) e_{1}+\left(z_{1}+i_{1} z_{2}\right) e_{2}\right\},
$$

for $\left(z_{1}-i_{1} z_{2}, z_{1}+i_{1} z_{2}\right) \in \mathbb{U}_{1} \times \mathbb{U}_{2}$. Here, the complex spaces $A_{1}, A_{2}$ are given as $A_{1}=$ $\left\{z_{1}-i_{1} z_{2}: z_{1}, z_{2} \in \mathbb{C}\right\}$ and $A_{2}=\left\{z_{1}+i_{1} z_{2}: z_{1}, z_{2} \in \mathbb{C}\right\}$. Let $a=\left(\alpha+i_{2} \beta\right)$ for $\alpha, \beta \in \mathbb{C}$.

If,

$$
\mathbb{U}_{1}=\left\{z_{1}-i_{1} z_{2} \in A_{1}:\left|z_{1}-i_{1} z_{2}-\left(\alpha-i_{1} \beta\right)\right|<r_{1}\right\}
$$

and,

$$
\mathbb{U}_{2}=\left\{z_{1}+i_{1} z_{2} \in A_{2}:\left|z_{1}+i_{1} z_{2}-\left(\alpha+i_{1} \beta\right)\right|<r_{2}\right\}
$$

then $\mathbb{U}$ in (6) is called the open discus with center $a$ and radii $r_{1}$ and $r_{2}$ and denoted by $\mathbf{D}\left(a ; r_{1}, r_{2}\right)$. If

$$
\mathbb{U}_{1}=\left\{z_{1}-i_{1} z_{2} \in A_{1}:\left|z_{1}-i_{1} z_{2}-\left(\alpha-i_{1} \beta\right)\right| \leq r_{1}\right\}
$$

and,

$$
\mathbb{U}_{2}=\left\{z_{1}+i_{1} z_{2} \in A_{2}:\left|z_{1}+i_{1} z_{2}-\left(\alpha+i_{1} \beta\right)\right| \leq r_{2}\right\}
$$


then $\mathbb{U}$ in (6) is called the closed discus with center $a$ and radii $r_{1}$ and $r_{2}$ and denoted by $\overline{\mathbf{D}}\left(a ; r_{1}, r_{2}\right)$. Thus,

$$
\begin{aligned}
\mathbf{D}\left(a ; r_{1}, r_{2}\right)= & \left\{z_{1}+i_{2} z_{2} \in \mathbb{C}_{2}:\right. \\
& z_{1}+i_{2} z_{2}=\left(z_{1}-i_{1} z_{2}\right) e_{1}+\left(z_{1}+i_{1} z_{2}\right) e_{2}, \\
& \left.\left|z_{1}-i_{1} z_{2}-\left(\alpha-i_{1} \beta\right)\right|<r_{1},\left|z_{1}+i_{1} z_{2}-\left(\alpha+i_{1} \beta\right)\right|<r_{2}\right\}
\end{aligned}
$$

and,

$$
\begin{aligned}
\overline{\mathbf{D}}\left(a ; r_{1}, r_{2}\right)= & \left\{z_{1}+i_{2} z_{2} \in \mathbb{C}_{2}:\right. \\
& z_{1}+i_{2} z_{2}=\left(z_{1}-i_{1} z_{2}\right) e_{1}+\left(z_{1}+i_{1} z_{2}\right) e_{2}, \\
& \left.\left|z_{1}-i_{1} z_{2}-\left(\alpha-i_{1} \beta\right)\right| \leq r_{1},\left|z_{1}+i_{1} z_{2}-\left(\alpha+i_{1} \beta\right)\right| \leq r_{2}\right\} .
\end{aligned}
$$

Let $\mathbf{D}$ be an open discus in $\mathbb{C}_{2}$. If taking the $\mathbb{C}_{2}$-cartesian product of two disks with center at $z_{1}+i_{2} z_{2}$ and radii $r_{1}$ and $r_{2}$ in $\mathbb{C}$, then it is defined by the following:

$$
\mathbf{D}\left(a ; r_{1}, r_{2}\right)=\mathbf{B}\left(\alpha-i_{1} \beta ; r_{1}\right) \times \mathbf{B}\left(\alpha+i_{1} \beta ; r_{2}\right)
$$

and,

$$
\overline{\mathbf{D}}\left(a ; r_{1}, r_{2}\right)=\overline{\mathbf{B}\left(\alpha-i_{1} \beta ; r_{1}\right)} \times \overline{\mathbf{B}\left(\alpha+i_{1} \beta ; r_{2}\right)} \subset \overline{\mathbf{D}\left(a ; r_{1}, r_{2}\right)} .
$$

For $a=0$, we can write:

$$
\begin{array}{r}
\mathbf{D}\left(0 ; r_{1}, r_{2}\right)=\mathbf{B}\left(0 ; r_{1}\right) \times \mathbf{B}\left(0 ; r_{2}\right) \\
=\left\{z_{1}+i_{2} z_{2} \in \mathbb{C}_{2}: z_{1}+i_{2} z_{2}=\left(z_{1}-i_{1} z_{2}\right) e_{1}+\left(z_{1}+i_{1} z_{2}\right) e_{2}\right. \\
\left.\left|z_{1}-i_{1} z_{2}\right| \leq r_{1},\left|z_{1}+i_{1} z_{2}\right| \leq r_{2}\right\}
\end{array}
$$

(see $[17,30])$.

Theorem 1 (see [17]). The idempotent component series of the bicomplex power series for all $k \in \mathbb{N}, a_{k}, b_{k}, z_{1}, z_{2} \in \mathbb{C}, \sum_{k=0}^{\infty}\left(a_{k}+i_{2} b_{k}\right)\left(z_{1}+i_{2} z_{2}\right)^{k}$ are the complex power series:

$$
\begin{aligned}
\sum_{k=0}^{\infty}\left(a_{k}+i_{2} b_{k}\right)\left(z_{1}+i_{2} z_{2}\right)^{k}= & {\left[\sum_{k=0}^{\infty}\left(a_{k}-i_{1} b_{k}\right)\left(z_{1}-i_{1} z_{2}\right)^{k}\right] e_{1} } \\
& +\left[\sum_{k=0}^{\infty}\left(a_{k}+i_{1} b_{k}\right)\left(z_{1}+i_{1} z_{2}\right)^{k}\right] e_{2} .
\end{aligned}
$$

Furthermore, let $f$ be a function defined by a power series as follows:

$$
f\left(z_{1}+i_{2} z_{2}\right)=\sum_{k=0}^{\infty}\left(a_{k}+i_{2} b_{k}\right)\left(z_{1}+i_{2} z_{2}\right)^{k}
$$

for $\left(z_{1}+i_{2} z_{2}\right) \in \mathbf{D}\left(0 ; r_{1}, r_{2}\right), r_{1}>0, r_{2}>0$. Define two complex-valued functions $f_{1}\left(z_{1}-i_{1} z_{2}\right)$, $f_{2}\left(z_{1}+i_{1} z_{2}\right) \in \mathbb{C}$ on the circles of convergence of the idempotent component power series as follows:

$$
\begin{array}{ll}
f_{1}\left(z_{1}-i_{1} z_{2}\right)=\sum_{k=0}^{\infty}\left(a_{k}-i_{1} b_{k}\right)\left(z_{1}-i_{1} z_{2}\right)^{k}, & \left|z_{1}-i_{1} z_{2}\right|<r_{1}, \\
f_{2}\left(z_{1}+i_{1} z_{2}\right)=\sum_{k=0}^{\infty}\left(a_{k}+i_{1} b_{k}\right)\left(z_{1}+i_{1} z_{2}\right)^{k}, & \left|z_{1}+i_{1} z_{2}\right|<r_{2} .
\end{array}
$$

In that case,

$$
f\left(z_{1}+i_{2} z_{2}\right)=f_{1}\left(z_{1}-i_{1} z_{2}\right) e_{1}+f_{2}\left(z_{1}+i_{1} z_{2}\right) e_{2} .
$$




\section{Some Approximation Properties of Bicomplex Bernstein Polynomials}

In this section, we define bicomplex Bernstein polynomials and obtain a quantitative upper estimate for the bicomplex Bernstein polynomials and its derivatives on a compact $\mathbb{C}_{2}$-disk. Later, we prove the qualitative Voronovskaja-type result for these polynomials.

Here, our goal is to introduce Bernstein polynomials for bicomplex numbers inspired by Equation (1).

Definition 2. If $f: \mathbf{D}\left(0 ; r_{1}, r_{2}\right) \rightarrow \mathbb{C}_{2}$ is a function, $n$ is a positive integer, $k=0,1,2, \ldots, n$, and then for $z_{1}+i_{2} z_{2} \in \mathbb{C}_{2}$, bicomplex Bernstein polynomials $B_{n}($.$) of f$ are defined as follows:

$$
\left(B_{n} f\right)\left(z_{1}+i_{2} z_{2}\right)=\sum_{k=0}^{n}\left(\begin{array}{l}
n \\
k
\end{array}\right)\left(z_{1}+i_{2} z_{2}\right)^{k}\left[1-\left(z_{1}+i_{2} z_{2}\right)\right]^{n-k} f\left(\frac{k}{n}\right) .
$$

Before giving some approximation theorems for the bicomplex Bernstein polynomials defined in Equation (10), we first need the following auxiliary results.

Lemma 1. If we denote $e_{k}\left(z_{1}+i_{2} z_{2}\right)=\left(z_{1}+i_{2} z_{2}\right)^{k}, \pi_{k, n}\left(z_{1}+i_{2} z_{2}\right)=\left(B_{n} e_{k}\right)\left(z_{1}+i_{2} z_{2}\right)$ then the recurrence formula:

$$
\begin{aligned}
\pi_{k+1, n}\left(z_{1}+i_{2} z_{2}\right) & =\frac{\left(z_{1}+i_{2} z_{2}\right)\left(1-\left(z_{1}+i_{2} z_{2}\right)\right)}{n} D_{z_{1}+i_{2} z_{2}}\left(\pi_{k, n}\right)\left(z_{1}+i_{2} z_{2}\right) \\
& +\left(z_{1}+i_{2} z_{2}\right) \pi_{k, n}\left(z_{1}+i_{2} z_{2}\right),
\end{aligned}
$$

holds for all $n \in \mathbb{N},\left(z_{1}+i_{2} z_{2}\right) \in \mathbb{C}_{2}$ and $k=0,1,2 \ldots$

Proof. Clearly, for $k=0,1,2$, we have $\pi_{0, n}\left(z_{1}+i_{2} z_{2}\right)=1, \pi_{1, n}\left(z_{1}+i_{2} z_{2}\right)=z_{1}+i_{2} z_{2}$ and $\pi_{2, n}\left(z_{1}+i_{2} z_{2}\right)=\left(z_{1}+i_{2} z_{2}\right)^{2}+\frac{\left(z_{1}+i_{2} z_{2}\right)-\left(z_{1}+i_{2} z_{2}\right)^{2}}{n}$.

Moreover, for any $k$, we get:

$$
\begin{aligned}
\pi_{k, n}\left(z_{1}+i_{2} z_{2}\right) & =\left(B_{n} e_{k}\right)\left(z_{1}+i_{2} z_{2}\right) \\
& =\left(\sum_{j=0}^{n}\left(\begin{array}{c}
n \\
j
\end{array}\right)\left(z_{1}-i_{1} z_{2}\right)^{j}\left[1-\left(z_{1}-i_{1} z_{2}\right)\right]^{n-j} \frac{j^{k}}{n^{k}}\right) e_{1} \\
& +\left(\sum_{j=0}^{n}\left(\begin{array}{c}
n \\
j
\end{array}\right)\left(z_{1}+i_{1} z_{2}\right)^{j}\left[1-\left(z_{1}+i_{1} z_{2}\right)\right]^{n-j} \frac{j^{k}}{n^{k}}\right) e_{2} .
\end{aligned}
$$

Therefore, for $n \in \mathbb{N}$ and $k=0,1, \ldots$, we can write:

$$
\begin{aligned}
D_{z_{1}+i_{2} z_{2}}\left(\pi_{k, n}\right)\left(z_{1}+i_{2} z_{2}\right) & =D_{z_{1}-i_{1} z_{2}}\left[\left(\sum_{j=0}^{n}\left(\begin{array}{c}
n \\
j
\end{array}\right)\left(z_{1}-i_{1} z_{2}\right)^{j}\right.\right. \\
& \left.\left.\times\left[1-\left(z_{1}-i_{1} z_{2}\right)\right]^{n-j} \frac{j^{k}}{n^{k}}\right) e_{1}\right] \\
& +D_{z_{1}+i_{1} z_{2}}\left[\left(\sum_{j=0}^{n}\left(\begin{array}{c}
n \\
j
\end{array}\right)\left(z_{1}+i_{1} z_{2}\right)^{j}\right.\right. \\
& \left.\left.\times\left[1-\left(z_{1}+i_{1} z_{2}\right)\right]^{n-j} \frac{j^{k}}{n^{k}}\right) e_{2}\right]
\end{aligned}
$$

and by a simple calculation, we obtain the following relationship: 


$$
\begin{aligned}
D_{z_{1}+i_{2} z_{2}}\left(\pi_{k, n}\right)\left(z_{1}+i_{2} z_{2}\right) & =\left(\frac{n}{\left(z_{1}-i_{1} z_{2}\right)\left(1-\left(z_{1}-i_{1} z_{2}\right)\right)} \pi_{k+1, n}\left(z_{1}-i_{1} z_{2}\right) e_{1}\right. \\
& \left.+\frac{n}{\left(z_{1}+i_{1} z_{2}\right)\left(1-\left(z_{1}+i_{1} z_{2}\right)\right)} \pi_{k+1, n}\left(z_{1}+i_{1} z_{2}\right) e_{2}\right) \\
& -\left(\frac{n}{1-\left(z_{1}-i_{1} z_{2}\right)} \pi_{k, n}\left(z_{1}-i_{1} z_{2}\right) e_{1}\right. \\
& \left.+\frac{n}{1-\left(z_{1}+i_{1} z_{2}\right)} \pi_{k, n}\left(z_{1}+i_{1} z_{2}\right) e_{2}\right) \\
& =\frac{n}{\left(z_{1}+i_{2} z_{2}\right)\left(1-\left(z_{1}+i_{2} z_{2}\right)\right)} \pi_{k+1, n}\left(z_{1}+i_{2} z_{2}\right) \\
& -\frac{n}{1-\left(z_{1}+i_{2} z_{2}\right)} \pi_{k, n}\left(z_{1}+i_{2} z_{2}\right) .
\end{aligned}
$$

Therefore, we get:

$$
\begin{aligned}
\pi_{k+1, n}\left(z_{1}+i_{2} z_{2}\right) & =\frac{\left(z_{1}+i_{2} z_{2}\right)\left(1-\left(z_{1}+i_{2} z_{2}\right)\right)}{n} D_{z_{1}+i_{2} z_{2}}\left(\pi_{k, n}\right)\left(z_{1}+i_{2} z_{2}\right) \\
& +\left(z_{1}+i_{2} z_{2}\right) \pi_{k, n}\left(z_{1}+i_{2} z_{2}\right),
\end{aligned}
$$

for all $\left(z_{1}+i_{2} z_{2}\right) \in \mathbb{C}_{2}, k=0,1, \ldots$ and $n \in \mathbb{N}$.

Lemma 2. Let $e_{k}$ and $\pi_{k, n}$ be defined as in Lemma 1 for all $n \in \mathbb{N},\left(z_{1}+i_{2} z_{2}\right) \in \mathbb{C}_{2}$ and $k=0,1,2 \ldots$.

If,

$$
\begin{aligned}
E_{k, n}\left(z_{1}+i_{2} z_{2}\right) & =\pi_{k, n}\left(z_{1}+i_{2} z_{2}\right)-e_{k}\left(z_{1}+i_{2} z_{2}\right) \\
& -\frac{\left(z_{1}+i_{2} z_{2}\right)^{k-1}\left(1-\left(z_{1}+i_{2} z_{2}\right)\right) k(k-1)}{2 n},
\end{aligned}
$$

then,

$$
\begin{aligned}
\left\|E_{k, n}\right\| & \leq\left\|E_{k-1, n}\right\|+\frac{\left\|z_{1}+i_{2} z_{2}\right\|\left\|1-\left(z_{1}+i_{2} z_{2}\right)\right\|}{2 n} \\
& \times\left\{2(k-1)\left\|\pi_{k-1, n}-e_{k-1}\right\|\right. \\
& \left.+2(k-1)\left\|\frac{(k-1)(k-2)\left(e_{k-2}-e_{k-1}\right)}{2 n}\right\|+\frac{2 k(k-1)(k-2)}{n}\right\}
\end{aligned}
$$

holds for all $n \in \mathbb{N},\left(z_{1}+i_{2} z_{2}\right) \in \mathbb{C}_{2}$ and $k \geq 2$.

Proof. From Equation (11), by replacing $k$ with $k-1$, we can write:

$$
\begin{aligned}
E_{k-1, n}\left(z_{1}+i_{2} z_{2}\right) & =\pi_{k-1, n}\left(z_{1}+i_{2} z_{2}\right)-e_{k-1}\left(z_{1}+i_{2} z_{2}\right) \\
& -\frac{\left(z_{1}+i_{2} z_{2}\right)^{k-2}\left(1-\left(z_{1}+i_{2} z_{2}\right)\right)(k-1)(k-2)}{2 n} .
\end{aligned}
$$

If the reduction relation, which is used in the previous Lemma 1 , then for all $\left(z_{1}+i_{2} z_{2}\right) \in$ $\mathbb{C}_{2}, n \in \mathbb{N}$, and $k \geq 2$ the following equation is provided: 


$$
\begin{aligned}
E_{k, n}\left(z_{1}+i_{2} z_{2}\right) & =\frac{\left(z_{1}+i_{2} z_{2}\right)\left(1-\left(z_{1}+i_{2} z_{2}\right)\right)}{n} \\
& \times D_{z_{1}+i_{2} z_{2}}\left(\pi_{k-1, n}\left(z_{1}+i_{2} z_{2}\right)-e_{k-1}\left(z_{1}+i_{2} z_{2}\right)\right) \\
& +\frac{(k-1)\left(z_{1}+i_{2} z_{2}\right)^{k-1}}{n}\left(1-\left(z_{1}+i_{2} z_{2}\right)\right) \\
& +\left(z_{1}+i_{2} z_{2}\right)\left[\pi_{k-1, n}\left(z_{1}+i_{2} z_{2}\right)-e_{k-1}\left(z_{1}+i_{2} z_{2}\right)\right] \\
& -\frac{\left(z_{1}+i_{2} z_{2}\right)^{k-1}\left(1-\left(z_{1}+i_{2} z_{2}\right)\right) k(k-1)}{2 n} \\
& =\frac{\left(z_{1}+i_{2} z_{2}\right)\left(1-\left(z_{1}+i_{2} z_{2}\right)\right)}{n} D_{z_{1}+i_{2} z_{2}} E_{k-1, n}\left(z_{1}+i_{2} z_{2}\right) \\
& +\left(z_{1}+i_{2} z_{2}\right) E_{k-1, n}\left(z_{1}+i_{2} z_{2}\right) \\
& +\frac{\left(z_{1}+i_{2} z_{2}\right)^{k-2}\left(1-\left(z_{1}+i_{2} z_{2}\right)\right)(k-1)(k-2)}{2 n^{2}} \\
& \times\left[(k-2)-\left(z_{1}+i_{2} z_{2}\right)(k-1)\right] .
\end{aligned}
$$

According to the Bernstein's inequality $\left\|D_{z_{1}+i_{2} z_{2}} E_{k-1, n}\right\| \leq(k-1)\left\|E_{k-1, n}\right\|$, for all $\left\|z_{1}+i_{2} z_{2}\right\| \leq 1, k \geq 2$ and $n \in \mathbb{N}$ (see [10,11]), we have:

$$
\begin{aligned}
\left\|E_{k, n}\right\| & \leq \frac{\left\|z_{1}+i_{2} z_{2}\right\|\left\|1-\left(z_{1}+i_{2} z_{2}\right)\right\|}{2 n}\left[2\left\|D_{z_{1}+i_{2} z_{2}} E_{k-1, n}\right\|\right] \\
& +\left\|E_{k-1, n}\right\|+\frac{\left\|z_{1}+i_{2} z_{2}\right\|\left\|1-\left(z_{1}+i_{2} z_{2}\right)\right\|}{2 n} \\
& \times \frac{\left\|z_{1}+i_{2} z_{2}\right\|^{k-3}(k-1)(k-2)}{n}(2 k-3) \\
& \leq\left\|E_{k-1, n}\right\|+\frac{\left\|z_{1}+i_{2} z_{2}\right\|\left\|1-\left(z_{1}+i_{2} z_{2}\right)\right\|}{2 n} \\
& \times\left\{2(k-1)\left\|\pi_{k-1, n}-e_{k-1}\right\|+2(k-1)\left\|\frac{(k-1)(k-2)\left(e_{k-2}-e_{k-1}\right)}{2 n}\right\|\right. \\
& \left.+\frac{2 k(k-1)(k-2)}{n}\right\} .
\end{aligned}
$$

Our first main result is the following theorem on the existence of a finite upper bound.

Theorem 2. Suppose that $R_{1}, R_{2}>1$ and $f: \mathbf{D}\left(0 ; R_{1}, R_{2}\right) \rightarrow \mathbb{C}_{2}$ is analytic in $\mathbf{D}\left(0 ; R_{1}, R_{2}\right)$, i.e.,

$$
f\left(z_{1}+i_{2} z_{2}\right)=\sum_{k=0}^{\infty}\left(a_{k}+i_{2} b_{k}\right)\left(z_{1}+i_{2} z_{2}\right)^{k}
$$

for all $\left(z_{1}+i_{2} z_{2}\right) \in \mathbf{D}\left(0 ; R_{1}, R_{2}\right)$.

(i) Let $r_{1}, r_{2}$ be arbitrary constants satisfying the inequalities $1 \leq r_{1}<R_{1}$ and $1 \leq r_{2}<R_{2}$. For all $\left\|z_{1}-i_{1} z_{2}\right\|_{r_{1}} \leq r_{1},\left\|z_{1}+i_{1} z_{2}\right\|_{r_{2}} \leq r_{2}$ and $n \in \mathbb{N}$, we get:

$$
\left\|\left(B_{n} f\right)-f\right\| \leq \frac{C_{r_{1}}(f)}{n}+\frac{C_{r_{2}}(f)}{n},
$$

where:

$$
0<C_{r_{1}}(f)=\frac{3 r_{1}\left(1+r_{1}\right)}{2} \sum_{j=2}^{\infty} j(j-1)\left|a_{j}-i_{1} b_{j}\right| r_{1}^{j-2}<\infty
$$


and:

$$
0<C_{r_{2}}(f)=\frac{3 r_{2}\left(1+r_{2}\right)}{2} \sum_{j=2}^{\infty} j(j-1)\left|a_{j}+i_{1} b_{j}\right| r_{2}^{j-2}<\infty .
$$

(ii) If $1 \leq r_{1}<r_{1}^{*}<R_{1}$ and $1 \leq r_{2}<r_{2}^{*}<R_{2}$ are arbitrary constants, then for all $\left\|z_{1}-i_{1} z_{2}\right\|_{r_{1}} \leq r_{1},\left\|z_{1}+i_{1} z_{2}\right\|_{r_{2}} \leq r_{2}$ and $n, p \in \mathbb{N}$,

$$
\left\|D_{z_{1}+i_{2} z_{2}}^{p}\left(B_{n} f\right)-D_{z_{1}+i_{2} z_{2}}^{p}(f)\right\| \leq \frac{p !}{n}\left\{\frac{C_{r_{1}}(f) r_{1}^{*}}{\left(r_{1}^{*}-r_{1}\right)^{p+1}}+\frac{C_{r_{2}}(f) r_{2}^{*}}{\left(r_{2}^{*}-r_{2}\right)^{p+1}}\right\},
$$

where $C_{r_{1}}(f)$ and $C_{r_{2}}(f)$ are given as in $(i)$.

Proof. See Appendix A.

Now, we are ready to consider the following Voronovskaja-type result for bicomplex Bernstein polynomials.

Theorem 3. Suppose that $R_{1}, R_{2}>1$ and $f: \mathbf{D}\left(0 ; R_{1}, R_{2}\right) \rightarrow \mathbb{C}_{2}$ is analytic in $\mathbf{D}\left(0 ; R_{1}, R_{2}\right)$, i.e.,

$$
f\left(z_{1}+i_{2} z_{2}\right)=\sum_{k=0}^{\infty}\left(c_{k}+i_{2} d_{k}\right)\left(z_{1}+i_{2} z_{2}\right)^{k}
$$

for all $\left(z_{1}+i_{2} z_{2}\right) \in \mathbf{D}\left(0 ; R_{1}, R_{2}\right)$. For all $\left(z_{1}+i_{2} z_{2}\right) \in \overline{\mathbf{D}}(0 ; 1,1)$ and $n \in \mathbb{N}$, the following Voronovskaja-type result in the closed unit disk holds as:

$$
\begin{aligned}
& \left|\left(B_{n} f\right)\left(z_{1}+i_{2} z_{2}\right)-f\left(z_{1}+i_{2} z_{2}\right)-\frac{\left(z_{1}+i_{2} z_{2}\right)\left(1-\left(z_{1}+i_{2} z_{2}\right)\right)}{2 n} D_{z_{1}+i_{2} z_{2}}^{2}(f)\right| \\
& \leq \frac{\left\|z_{1}+i_{2} z_{2}\right\|\left\|1-\left(z_{1}+i_{2} z_{2}\right)\right\|}{2 n} \frac{18}{n} M(f),
\end{aligned}
$$

where $0<M(f)=\sum_{k=3}^{\infty}\left|c_{k}+i_{2} d_{k}\right| k(k-1)(k-2)^{2}<\infty$.

Proof. From the proof of Theorem $2(i)$, for every $n \in \mathbb{N}$, we evidently have:

$$
\begin{aligned}
& \left|\left(B_{n} f\right)\left(z_{1}+i_{2} z_{2}\right)-f\left(z_{1}+i_{2} z_{2}\right)-\frac{\left(z_{1}+i_{2} z_{2}\right)\left(1-\left(z_{1}+i_{2} z_{2}\right)\right)}{2 n} D_{z_{1}+i_{2} z_{2}}^{2}(f)\right| \\
& =\mid \sum_{k=0}^{\infty}\left(c_{k}+i_{2} d_{k}\right)\left(B_{n} e_{k}\right)\left(z_{1}+i_{2} z_{2}\right)-\sum_{k=0}^{\infty}\left(c_{k}+i_{2} d_{k}\right) e_{k}\left(z_{1}+i_{2} z_{2}\right) \\
& -\frac{\left(z_{1}+i_{2} z_{2}\right)\left(1-\left(z_{1}+i_{2} z_{2}\right)\right)}{2 n} \sum_{k=2}^{\infty}\left(c_{k}+i_{2} d_{k}\right) k(k-1)\left(z_{1}+i_{2} z_{2}\right)^{k-2} \mid \\
& \leq \sum_{k=3}^{\infty}\left|c_{k}+i_{2} d_{k}\right|\left|E_{k, n}\left(z_{1}+i_{2} z_{2}\right)\right|
\end{aligned}
$$


Furthermore, if $r_{1}=r_{2}=1$ is taken in the inequality obtained in the proof of Theorem $2(i)$, we get $\left\|\pi_{k, n}-e_{k}\right\| \leq \frac{6}{n} k(k-1)$. As a consequence, from Lemma 2, we can write:

$$
\begin{aligned}
\left\|E_{k, n}\right\| & \leq\left\|E_{k-1, n}\right\|+\frac{\left\|z_{1}+i_{2} z_{2}\right\|\left\|1-\left(z_{1}+i_{2} z_{2}\right)\right\|}{2 n} \\
& \times\left\{2(k-1) \frac{6}{n}(k-1)(k-2)\right. \\
& +2(k-1)\left\|\frac{(k-1)(k-2)}{2 n}\left(e_{k-2}-e_{k-1}\right)\right\| \\
& \left.+\frac{2 k(k-1)(k-2)}{n}\right\}
\end{aligned}
$$

and $\left\|e_{k-2}-e_{k-1}\right\| \leq r_{1}^{k-2}\left(1+r_{1}\right)+r_{2}^{k-2}\left(1+r_{2}\right) \leq 4$, for $r_{1}<1, r_{2}<1$, which immediately implies:

$$
\begin{aligned}
\left\|E_{k, n}\right\| & \leq\left\|E_{k-1, n}\right\|+\frac{\left\|z_{1}+i_{2} z_{2}\right\|\left\|1-\left(z_{1}+i_{2} z_{2}\right)\right\|}{2 n} \\
& \times \frac{18 k(k-1)(k-2)}{n} .
\end{aligned}
$$

Equation (11), yields $E_{0, n}\left(z_{1}+i_{2} z_{2}\right)=E_{1, n}\left(z_{1}+i_{2} z_{2}\right)=E_{2, n}\left(z_{1}+i_{2} z_{2}\right)=0$, for $k=0,1,2$. Therefore, by writing the above last inequality for $k=3,4, \ldots$, we clearly obtain, step by step the following:

$$
\begin{aligned}
\left\|E_{k, n}\right\| & \leq \frac{\left\|z_{1}+i_{2} z_{2}\right\|\left\|1-\left(z_{1}+i_{2} z_{2}\right)\right\|}{2 n} \\
& \times \frac{18}{n} \sum_{j=3}^{k} j(j-1)(j-2) \\
& \leq \frac{\left\|z_{1}+i_{2} z_{2}\right\|\left\|1-\left(z_{1}+i_{2} z_{2}\right)\right\|}{2 n} \frac{18}{n} k(k-1)(k-2)^{2} .
\end{aligned}
$$

Thus, we get:

$$
\begin{aligned}
& \left|\left(B_{n} f\right)\left(z_{1}+i_{2} z_{2}\right)-f\left(z_{1}+i_{2} z_{2}\right)-\frac{\left(z_{1}+i_{2} z_{2}\right)\left(1-\left(z_{1}+i_{2} z_{2}\right)\right)}{2 n} D_{z_{1}+i_{2} z_{2}}^{2}(f)\right| \\
& \leq\left\|\frac{\left(z_{1}+i_{2} z_{2}\right)\left(1-\left(z_{1}+i_{2} z_{2}\right)\right)}{2 n}\right\| \frac{18}{n} \sum_{k=3}^{\infty}\left|c_{k}+i_{2} d_{k}\right| k(k-1)(k-2)^{2} \\
& \leq\left\|\frac{\left(z_{1}+i_{2} z_{2}\right)\left(1-\left(z_{1}+i_{2} z_{2}\right)\right)}{2 n}\right\| \frac{18}{n} M(f) .
\end{aligned}
$$

Since:

$$
\begin{aligned}
D_{z_{1}+i_{2} z_{2}}^{4} f\left(z_{1}+i_{2} z_{2}\right) & =\left[\sum_{k=4}^{\infty}\left(c_{k}-i_{1} d_{k}\right) k(k-1)(k-2)(k-3)\left(z_{1}-i_{1} z_{2}\right)^{k-4}\right] e_{1} \\
& +\left[\sum_{k=4}^{\infty}\left(c_{k}+i_{1} d_{k}\right) k(k-1)(k-2)(k-3)\left(z_{1}+i_{1} z_{2}\right)^{k-4}\right] e_{2}
\end{aligned}
$$

and both series are absolutely convergent, we have:

$$
M(f)=\sum_{k=3}^{\infty}\left(c_{k}+i_{2} d_{k}\right) k(k-1)(k-2)^{2}<\infty .
$$

Therefore, Theorem 3 has been proved. 
Theorem 4. Suppose that $R_{1}, R_{2}>1$ and $f: \mathbf{D}\left(0 ; R_{1}, R_{2}\right) \rightarrow \mathbb{C}_{2}$ is analytic in $\mathbf{D}\left(0 ; R_{1}, R_{2}\right)$, i.e.,

$$
f\left(z_{1}+i_{2} z_{2}\right)=\sum_{k=0}^{\infty}\left(c_{k}+i_{2} d_{k}\right)\left(z_{1}+i_{2} z_{2}\right)^{k}
$$

for all $\left(z_{1}+i_{2} z_{2}\right) \in \mathbf{D}\left(0 ; R_{1}, R_{2}\right)$. Let $1 \leq r_{1}<R_{1}$ and $1 \leq r_{2}<R_{2}$. For all $n \in \mathbb{N}$, $\left|z_{1}-i_{1} z_{2}\right|<r_{1}$ and $\left|z_{1}+i_{1} z_{2}\right|<r_{2}$,

$$
\begin{aligned}
& \left|\left(B_{n} f\right)\left(z_{1}-i_{1} z_{2}\right)-f\left(z_{1}-i_{1} z_{2}\right)-\frac{\left(z_{1}-i_{1} z_{2}\right)\left(1-\left(z_{1}-i_{1} z_{2}\right)\right)}{2 n} D_{z_{1}-i_{1} z_{2}}^{2}(f)\right| \\
& \leq \frac{5\left(1+r_{1}\right)^{2}}{2 n} \frac{M_{r_{1}}(f)}{n}
\end{aligned}
$$

and,

$$
\begin{aligned}
& \left|\left(B_{n} f\right)\left(z_{1}+i_{1} z_{2}\right)-f\left(z_{1}+i_{1} z_{2}\right)-\frac{\left(z_{1}+i_{1} z_{2}\right)\left(1-\left(z_{1}+i_{1} z_{2}\right)\right)}{2 n} D_{z_{1}+i_{1} z_{2}}^{2}(f)\right| \\
& \leq \frac{5\left(1+r_{2}\right)^{2}}{2 n} \frac{M_{r_{2}}(f)}{n}
\end{aligned}
$$

where,

$M_{r_{1}}(f)=\sum_{k=3}^{\infty}\left|c_{k}-i_{1} d_{k}\right| k(k-1)(k-2)^{2} r_{1}^{k-2}<\infty$

and,

$M_{r_{2}}(f)=\sum_{k=3}^{\infty}\left|c_{k}+i_{1} d_{k}\right| k(k-1)(k-2)^{2} r_{1}^{k-2}<\infty$.

Proof. See Appendix B.

Theorem 5. Suppose that $r_{1}, r_{2}>1$ and $f: \mathbf{D}\left(0 ; r_{1}, r_{2}\right) \rightarrow \mathbb{C}_{2}$ is analytic in $\mathbf{D}\left(0 ; r_{1}, r_{2}\right)$. If $f$ is not a polynomial of degree $\leq 1$, then for every $r_{1}^{*} \in\left[1, r_{1}\right]$ and $r_{2}^{*} \in\left[1, r_{2}\right]$ the following are satisfied, for every $n \in \mathbb{N}$,

$$
\left\|B_{n} f-f\right\|_{r_{1}^{*}} \geq \frac{C_{r_{1}^{*}}(f)}{n} \text { and }\left\|B_{n} f-f\right\|_{r_{2}^{*}} \geq \frac{C_{r_{2}^{*}}(f)}{n}
$$

where $\|f\|_{r_{1}^{*}}=\max \left\{f\left(z_{1}-i_{1} z_{2}\right):\left|z_{1}-i_{1} z_{2}\right| \leq r_{1}^{*}\right\},\|f\|_{r_{2}^{*}}=\max \left\{f\left(z_{1}+i_{1} z_{2}\right):\left|z_{1}+i_{1} z_{2}\right|\right.$ $\left.\leq r_{2}^{*}\right\}$ and $\mathbf{D}_{r_{1}}=\left\{\left|z_{1}-i_{1} z_{2}\right|<r_{1}: z_{1}, z_{2} \in \mathbb{C}\right\}, \mathbf{D}_{r_{2}}=\left\{\left|z_{1}+i_{1} z_{2}\right|<r_{2}: z_{1}, z_{2} \in \mathbb{C}\right\}$. The constant $C_{r_{1}^{*}}(f)$ depends on function $f$ and $r_{1}^{*}$, while again the constant $C_{r_{2}^{*}}(f)$ depends on function $f$ and $r_{2}^{*}$.

Proof. See Appendix C.

Now, combining Theorem 5 with Theorem 2 (i), we immediately get the following Corollary 1.

Corollary 1. Suppose that $r_{1}, r_{2}>1$ and $f: \mathbf{D}\left(0 ; r_{1}, r_{2}\right) \rightarrow \mathbb{C}_{2}$ is analytic in $\mathbf{D}\left(0 ; r_{1}, r_{2}\right)$. If $f$ is not a polynomial of degree $\leq 1$, then for every $r_{1}^{*} \in\left[1, r_{1}\right)$ and $r_{2}^{*} \in\left[1, r_{2}\right)$ the following are satisfied. For every $n \in \mathbb{N}$, we have:

$$
\left\|B_{n} f-f\right\|_{r_{1}^{*}} \backsim \frac{1}{n} \text { and }\left\|B_{n} f-f\right\|_{r_{2}^{*}} \backsim \frac{1}{n} .
$$

\section{Conclusions}

In recent years, mostly the authors of mathematics and physics research have focused on bicomplex numbers and bicomplex analyses (see [13,31-33]). This trend will continue thanks to possible applications of bicomplex algebra in engineering and good results in approximation theory. In this paper, considering the advantages of this field, we have tried to carry relevant theoretical background and some generalizations to ap- 
proximation theory by means of operators in the sense of Bernstein polynomials with a similar manner to complex Bernstein polynomials. As further work, in addition to getting better approximation results, similar studies may be integrated into frames of other convenient polynomials or operators; application possibilities and much more links to the engineering like Hopfield neural networks, signal processing, etc., may be investigated (see [34-36]).

See the appendix below to examine the details of proofs of Theorems 2-5.

Author Contributions: Writing-original draft, C.C., Ç.A., Ö.Ö.G. and S.K.; Writing-review-editing, C.C., Ç.A., Ö.Ö.G. and S.K. All authors have read and agreed to the published version of the manuscript.

Funding: This research received no external funding.

Institutional Review Board Statement: Not applicable.

Informed Consent Statement: Not applicable.

Data Availability Statement: Not applicable.

Conflicts of Interest: The authors declare no conflict of interest.

\section{Appendix A. Proof of Theorem 2}

(i) Denoting $e_{k}\left(z_{1}+i_{2} z_{2}\right)=\left(z_{1}+i_{2} z_{2}\right)^{k}, \pi_{k, n}\left(z_{1}+i_{2} z_{2}\right)=\left(B_{n} e_{k}\right)\left(z_{1}+i_{2} z_{2}\right)$ for $k=0,1 \ldots$ and $\mathbf{D}\left(0 ; R_{1}, R_{2}\right)=\left\{z_{1}+i_{2} z_{2} \in \mathbb{C}_{2}: z_{1}+i_{2} z_{2}=\left(z_{1}-i_{1} z_{2}\right) e_{1}+\left(z_{1}+i_{1} z_{2}\right) e_{2}\right.$, $\left.\left|z_{1}-i_{1} z_{2}\right|<R_{1},\left|z_{1}+i_{1} z_{2}\right|<R_{2}\right\}$. Now, as in (6) by using the idempotent representation of $f\left(z_{1}+i_{2} z_{2}\right)$, we get following inequality. If we use the iteration relation (cited in [10]), then;

$$
\left(B_{n} f\right)\left(z_{1}+i_{2} z_{2}\right)=\sum_{k=0}^{\infty}\left(a_{k}+i_{2} b_{k}\right) \pi_{k, n}\left(z_{1}+i_{2} z_{2}\right)
$$

and we can write:

$$
\left\|\left(B_{n} f\right)-f\right\| \leq \sqrt{2} \sum_{k=0}^{\infty}\left\|a_{k}+i_{2} b_{k}\right\|\left\|\pi_{k, n}-e_{k}\right\|,
$$

for all $\left(z_{1}+i_{2} z_{2}\right) \in \mathbf{D}\left(0 ; R_{1}, R_{2}\right)$. So that we need an estimate for $\left\|\pi_{k, n}-e_{k}\right\|$. Firstly, by replacing $k$ with $k-1$ in Lemma 1 , then with simple calculation we get:

$$
\begin{aligned}
\pi_{k, n}\left(z_{1}+i_{2} z_{2}\right)-\left(z_{1}+i_{2} z_{2}\right)^{k} & =\frac{\left(z_{1}+i_{2} z_{2}\right)\left(1-\left(z_{1}+i_{2} z_{2}\right)\right)}{n} \\
& \times D_{z_{1}+i_{2} z_{2}}\left(\pi_{k-1, n}\left(z_{1}+i_{2} z_{2}\right)-\left(z_{1}+i_{2} z_{2}\right)^{k-1}\right) \\
& +\frac{(k-1)\left(z_{1}+i_{2} z_{2}\right)^{k-1}}{n}\left(1-\left(z_{1}+i_{2} z_{2}\right)\right) \\
& +\left(z_{1}+i_{2} z_{2}\right)\left[\pi_{k-1, n}\left(z_{1}+i_{2} z_{2}\right)-\left(z_{1}+i_{2} z_{2}\right)^{k-1}\right] .
\end{aligned}
$$

Let $\overline{\mathbf{D}}\left(0 ; r_{1}, r_{2}\right)=\left\{z_{1}+i_{2} z_{2} \in \mathbb{C}_{2}: z_{1}+i_{2} z_{2}=\left(z_{1}-i_{1} z_{2}\right) e_{1}+\left(z_{1}+i_{1} z_{2}\right) e_{2}, \mid z_{1}-\right.$ $\left.i_{1} z_{2}\left|\leq r_{1},\right| z_{1}+i_{1} z_{2} \mid \leq r_{2}\right\}$, for $r_{1} \geq 1, r_{2} \geq 1$. According to Bernstein's inequality (see [11]) in the closed unit disk, by a linear transformation, if $P_{k}$ represents an algebraic polynomial with a degree less than or equal to $k$ and also considering the Formula (7), we can write $\left\|D_{z_{1}+i_{2} z_{2}} P_{k}\right\| \leq \frac{k}{r_{1}}\left\|P_{k}\right\|_{r_{1}}+\frac{k}{r_{2}}\left\|P_{k}\right\|_{r_{2}}$ and from the above inequality, we obtain: 


$$
\begin{aligned}
\left\|\pi_{k, n}-e_{k}\right\| & \leq\left\{\frac{\left|z_{1}-i_{1} z_{2}\right|\left(1+\left|z_{1}-i_{1} z_{2}\right|\right)}{n} \frac{(k-1)}{r_{1}}\right. \\
& \times\left\|\pi_{k-1, n}-e_{k-1}\right\|_{r_{1}} \\
& +\frac{(k-1)\left|z_{1}-i_{1} z_{2}\right|\left(1+\left|z_{1}-i_{1} z_{2}\right|\right)}{n}+\left|z_{1}-i_{1} z_{2}\right| \\
& \left.\times\left|\pi_{k-1, n}\left(z_{1}-i_{1} z_{2}\right)-e_{k-1}\left(z_{1}-i_{1} z_{2}\right)\right|\right\}\left\|e_{1}\right\| \\
& +\left\{\frac{\left|z_{1}+i_{1} z_{2}\right|\left(1+\left|z_{1}+i_{1} z_{2}\right|\right)}{n} \frac{(k-1)}{r_{2}}\right. \\
& \times\left\|\pi_{k-1, n}-e_{k-1}\right\|_{r_{2}} \\
& +\frac{(k-1)\left|z_{1}+i_{1} z_{2}\right|\left(1+\left|z_{1}+i_{1} z_{2}\right|\right)}{n}+\left|z_{1}+i_{1} z_{2}\right| \\
& \left.\times\left|\pi_{k-1, n}\left(z_{1}+i_{1} z_{2}\right)-e_{k-1}\left(z_{1}+i_{1} z_{2}\right)\right|\right\}\left\|e_{2}\right\|,
\end{aligned}
$$

Since, $\left|z_{1}-i_{1} z_{2}\right| \leq r_{1}, r_{1} \geq 1,\left|z_{1}+i_{1} z_{2}\right| \leq r_{2}, r_{2} \geq 1$, we have $\left|\pi_{k, n}\left(z_{1}-i_{1} z_{2}\right)\right| \leq r_{1}^{k}$ and $\left|\pi_{k, n}\left(z_{1}+i_{1} z_{2}\right)\right| \leq r_{2}^{k}$, for every $k, n \in \mathbb{N}$, (see [3]). Thus, by using the last inequality, we easily obtain:

$$
\begin{aligned}
\left\|\pi_{k, n}-e_{k}\right\| & \leq r_{1}\left|\pi_{k-1, n}\left(z_{1}-i_{1} z_{2}\right)-e_{k-1}\left(z_{1}-i_{1} z_{2}\right)\right| \\
& +3\left(1+r_{1}\right) r_{1}^{k-1} \frac{(k-1)}{n} \\
& +r_{2}\left|\pi_{k-1, n}\left(z_{1}+i_{1} z_{2}\right)-e_{k-1}\left(z_{1}+i_{1} z_{2}\right)\right| \\
& +3\left(1+r_{2}\right) r_{2}^{k-1} \frac{(k-1)}{n} .
\end{aligned}
$$

Again, by using the reduction relation, we get:

$$
\begin{aligned}
\left\|\pi_{k, n}-e_{k}\right\| & \leq \frac{3\left(1+r_{1}\right)}{n}\left[r_{1}^{k-1}+2 r_{1}^{k-1}+\ldots+(k-1) r_{1}^{k-1}\right] \\
& +\frac{3\left(1+r_{2}\right)}{n}\left[r_{2}^{k-1}+2 r_{2}^{k-1}+\ldots+(k-1) r_{2}^{k-1}\right] \\
& \leq \frac{3\left(1+r_{1}\right)}{n} r_{1}^{k-1} \frac{(k-1) k}{2}+\frac{3\left(1+r_{2}\right)}{n} r_{2}^{k-1} \frac{(k-1) k}{2} \\
& =\frac{3 r_{1}\left(1+r_{1}\right)}{2 n} k(k-1) r_{1}^{k-2}+\frac{3 r_{2}\left(1+r_{2}\right)}{2 n} k(k-1) r_{2}^{k-2} .
\end{aligned}
$$

For all $\left(z_{1}+i_{2} z_{2}\right) \in \mathbf{D}\left(0 ; R_{1}, R_{2}\right), k=0,1, \ldots$ and $n \in \mathbb{N}$.

Note that from hypothesis, $f\left(z_{1}+i_{2} z_{2}\right)$ is absolutely and uniformly convergent in $\left|z_{1}-i_{1} z_{2}\right| \leq r_{1}$ and $\left|z_{1}+i_{1} z_{2}\right| \leq r_{2}$, respectively. Then, for $1 \leq r_{1}<R_{1}$ and $1 \leq r_{2}<R_{2}$, the power series on the right hand side can be differentiated twice:

$$
D_{z_{1}-i_{1} z_{2}}^{2} f_{1}\left(z_{1}-i_{1} z_{2}\right)=\sum_{k=2}^{\infty} k(k-1)\left(a_{k}-i_{1} b_{k}\right) \times\left(z_{1}-i_{1} z_{2}\right)^{k-2}
$$

and,

$$
D_{z_{1}+i_{1} z_{2}}^{2} f_{2}\left(z_{1}+i_{1} z_{2}\right)=\sum_{k=2}^{\infty} k(k-1)\left(a_{k}+i_{1} b_{k}\right)\left(z_{1}+i_{1} z_{2}\right)^{k-2}
$$

are also absolutely convergent.

Thus, $\sum_{k=2}^{\infty} k(k-1)\left|a_{k}-i_{1} b_{k}\right| r_{1}^{k-2}<\infty$ and $\sum_{k=2}^{\infty} k(k-1)\left|a_{k}+i_{1} b_{k}\right| r_{2}^{k-2}<\infty$.

(ii) By using Cauchy integral formula and Cauchy derivation formula for bicomplex functions (see [17]), we prove desired results as follows: 
Let $\gamma=\mathbf{D}\left(0 ; r_{1}^{*}, r_{2}^{*}\right)$ be an open ball with zero center and $r_{1}^{*}, r_{2}^{*}$ radii $\left(r_{1}^{*}>1, r_{2}^{*}>1\right)$ and $z_{1}+i_{2} z_{2}=\left(z_{1}-i_{1} z_{2}\right) e_{1}+\left(z_{1}+i_{1} z_{2}\right) e_{2}$, where $\left|z_{1}-i_{1} z_{2}\right|<r_{1}$ and $\left|z_{1}+i_{1} z_{2}\right|<r_{2}$. For $\left(\gamma_{1}+i_{2} \gamma_{2}\right) \in \gamma$,

$$
\begin{aligned}
\left\|\gamma_{1}+i_{2} \gamma_{2}-\left(z_{1}+i_{2} z_{2}\right)\right\| & \geq\left\|\gamma_{1}-i_{1} \gamma_{2}-\left(z_{1}-i_{1} z_{2}\right)\right\|-\left\|\gamma_{1}+i_{1} \gamma_{2}-\left(z_{1}+i_{1} z_{2}\right)\right\| \\
& \geq\left(r_{1}^{*}-r_{1}\right)-\left(r_{2}^{*}-r_{2}\right)
\end{aligned}
$$

is obtained. Now, a simple calculation leads us to the following result:

$$
\begin{aligned}
& \left\|D_{z_{1}+i_{2} z_{2}}^{p}\left(B_{n} f\right)-D_{z_{1}+i_{2} z_{2}}^{p}(f)\right\| \\
& \leq \frac{p !}{2 \pi}\left|\int_{\gamma_{1}} \frac{\left[\left(B_{n} f_{1}\right)\left(\gamma_{1}-i_{1} \gamma_{2}\right)-f_{1}\left(\gamma_{1}-i_{1} \gamma_{2}\right)\right]}{\left[\left(\gamma_{1}-i_{1} \gamma_{2}\right)-\left(z_{1}-i_{1} z_{2}\right)\right]^{p+1}} d\left(\gamma_{1}-i_{1} \gamma_{2}\right)\right| \\
& +\frac{p !}{2 \pi}\left|\int_{\gamma_{2}} \frac{\left[\left(B_{n} f_{2}\right)\left(\gamma_{1}+i_{1} \gamma_{2}\right)-f_{2}\left(\gamma_{1}+i_{1} \gamma_{2}\right)\right]}{\left[\left(\gamma_{1}+i_{1} \gamma_{2}\right)-\left(z_{1}+i_{1} z_{2}\right)\right]^{p+1}} d\left(\gamma_{1}+i_{1} \gamma_{2}\right)\right| \\
& =\frac{p !}{n}\left\{\frac{C_{r_{1}}(f) r_{1}^{*}}{\left(r_{1}^{*}-r_{1}\right)^{p+1}}+\frac{C_{r_{2}}(f) r_{2}^{*}}{\left(r_{2}^{*}-r_{2}\right)^{p+1}}\right\}
\end{aligned}
$$

and the proof is completed.

\section{Appendix B. Proof of Theorem 4}

Using Theorem 3, we obtain that for every $n \in \mathbb{N}$ and $k \geq 2$,

$$
\begin{aligned}
E_{k, n}\left(z_{1}+i_{2} z_{2}\right) & =\left(\frac{\left(z_{1}-i_{1} z_{2}\right)\left(1-\left(z_{1}-i_{1} z_{2}\right)\right)}{n} D_{z_{1}-i_{1} z_{2}} E_{k-1, n}\left(z_{1}-i_{1} z_{2}\right)\right. \\
& +\left(z_{1}-i_{1} z_{2}\right) E_{k-1, n}\left(z_{1}-i_{1} z_{2}\right) \\
& +\left[(k-2)-\left(z_{1}-i_{1} z_{2}\right)(k-1)\right] \\
& \left.\times \frac{\left(z_{1}-i_{1} z_{2}\right)^{k-2}\left(1-\left(z_{1}-i_{1} z_{2}\right)\right)(k-1)(k-2)}{2 n^{2}}\right) e_{1} \\
& +\left(\frac{\left(z_{1}+i_{1} z_{2}\right)\left(1-\left(z_{1}+i_{1} z_{2}\right)\right)}{n} D_{z_{1}+i_{1} z_{2}} E_{k-1, n}\left(z_{1}+i_{1} z_{2}\right)\right. \\
& +\left(z_{1}+i_{1} z_{2}\right) E_{k-1, n}\left(z_{1}+i_{1} z_{2}\right) \\
& +\left[(k-2)-\left(z_{1}+i_{1} z_{2}\right)(k-1)\right] \\
& \left.\times \frac{\left(z_{1}+i_{1} z_{2}\right)^{k-2}\left(1-\left(z_{1}+i_{1} z_{2}\right)\right)(k-1)(k-2)}{2 n^{2}}\right) e_{2} .
\end{aligned}
$$

So, for all $k, n \in \mathbb{N},\left|z_{1}-i_{1} z_{2}\right| \leq r_{1}$ and $\left|z_{1}+i_{1} z_{2}\right| \leq r_{2}, r_{1}, r_{2} \geq 1$, we can write:

$$
\begin{aligned}
\left|E_{k, n}\left(z_{1}+i_{2} z_{2}\right)\right| & \leq\left(\frac{r_{1}\left(1+r_{1}\right)}{n}\left|D_{z_{1}-i_{1} z_{2}} E_{k-1, n}\left(z_{1}-i_{1} z_{2}\right)\right|\right. \\
& +r_{1}\left|E_{k-1, n}\left(z_{1}-i_{1} z_{2}\right)\right| \\
& \left.+\frac{\left(1+r_{1}\right) r_{1}^{k-2}(k-1)(k-2)}{2 n^{2}}\left[(k-2)+r_{1}(k-1)\right]\right) \\
& +\left(\frac{r_{2}\left(1+r_{2}\right)}{n}\left|D_{z_{1}+i_{1} z_{2}} E_{k-1, n}\left(z_{1}+i_{1} z_{2}\right)\right|\right. \\
& +r_{2}\left|E_{k-1, n}\left(z_{1}+i_{1} z_{2}\right)\right| \\
& \left.+\frac{\left(1+r_{2}\right) r_{2}^{k-2}(k-1)(k-2)}{2 n^{2}}\left[(k-2)+r_{2}(k-1)\right]\right) .
\end{aligned}
$$


Now, we calculate the following for $k \geq 3$,

$$
\begin{aligned}
\left|D_{z_{1}+i_{2} z_{2}} E_{k-1, n}\left(z_{1}+i_{2} z_{2}\right)\right| & \leq\left|D_{z_{1}-i_{1} z_{2}} E_{k-1, n}\left(z_{1}-i_{1} z_{2}\right)\right| \\
& +\left|D_{z_{1}+i_{1} z_{2}} E_{k-1, n}\left(z_{1}+i_{1} z_{2}\right)\right| \\
& \leq \frac{k-1}{r_{1}}\left\|E_{k-1, n}\right\|_{r_{1}} \\
& +\frac{k-1}{r_{2}}\left\|E_{k-1, n}\right\|_{r_{2}} .
\end{aligned}
$$

and from Equation (12), we have:

$$
\begin{aligned}
E_{k-1, n}\left(z_{1}+i_{2} z_{2}\right) & =\pi_{k-1, n}\left(z_{1}+i_{2} z_{2}\right)-e_{k-1}\left(z_{1}+i_{2} z_{2}\right) \\
& -\frac{(k-1)(k-2)}{2 n}\left(e_{k-2}\left(z_{1}+i_{2} z_{2}\right)-e_{k-1}\left(z_{1}+i_{2} z_{2}\right)\right) .
\end{aligned}
$$

If we employe the following relation which is used in Theorem 3 for all $k, n \in \mathbb{N}, r_{1}$, $r_{2} \geq 1,\left\|z_{1}-i_{1} z_{2}\right\|_{r_{1}} \leq r_{1}$ and $\left\|z_{1}+i_{1} z_{2}\right\|_{r_{2}} \leq r_{2}$

$$
\left\|\pi_{k, n}-e_{k}\right\| \leq \frac{3 r_{1}\left(1+r_{1}\right)}{2 n} k(k-1) r_{1}^{k-2}+\frac{3 r_{2}\left(1+r_{2}\right)}{2 n} k(k-1) r_{2}^{k-2} .
$$

Then, for every $n \in \mathbb{N}, k \geq 2$, we clearly obtain the following inequality,

$$
\begin{array}{r}
\left|D_{z_{1}+i_{2} z_{2}} E_{k-1, n}\left(z_{1}+i_{2} z_{2}\right)\right| \leq \\
+\frac{k-1}{r_{1}}\left\{\left\|\pi_{k-1, n}-e_{k-1}\right\|_{r_{1}}+\left\|\frac{(k-1)(k-2)\left(e_{k-1}-e_{k-2}\right)}{2 n}\right\|_{r_{1}}\right\} \\
+\frac{k-1}{r_{2}}\left\{\left\|\pi_{k-1, n}-e_{k-1}\right\|_{r_{2}}+\left\|\frac{(k-1)(k-2)\left(e_{k-1}-e_{k-2}\right)}{2 n}\right\|_{r_{2}}\right\} \\
=\frac{2 k(k-1)(k-2)}{n}\left(r_{1}^{k-3}\left(1+r_{1}\right)+r_{2}^{k-3}\left(1+r_{2}\right)\right) .
\end{array}
$$

This implies:

$$
\frac{r_{1}\left(1+r_{1}\right)}{n}\left|D_{z_{1}-i_{1} z_{2}} E_{k-1, n}\left(z_{1}-i_{1} z_{2}\right)\right| \leq \frac{2 r_{1}\left(1+r_{1}\right)^{2} k(k-1)(k-2) r_{1}^{k-3}}{n^{2}}
$$

and,

$$
\frac{r_{2}\left(1+r_{2}\right)}{n}\left|D_{z_{1}+i_{1} z_{2}} E_{k-1, n}\left(z_{1}+i_{1} z_{2}\right)\right| \leq \frac{2 r_{2}\left(1+r_{2}\right)^{2} k(k-1)(k-2) r_{2}^{k-3}}{n^{2}} .
$$

Thus, from the last two inequalities above, we can write:

$$
\begin{aligned}
\left|E_{k, n}\left(z_{1}-i_{1} z_{2}\right)\right| & \leq \frac{2 r_{1}\left(1+r_{1}\right)^{2} k(k-1)(k-2) r_{1}^{k-3}}{n^{2}} \\
& +r_{1}\left|E_{k-1, n}\left(z_{1}-i_{1} z_{2}\right)\right| \\
& +\frac{\left(1+r_{1}\right)(k-1)(k-2) r_{1}^{k-2}}{2 n^{2}}\left[(k-2)+r_{1}(k-1)\right] \\
& =r_{1}\left|E_{k-1, n}\left(z_{1}-i_{1} z_{2}\right)\right| \\
& +\frac{\left(1+r_{1}\right)(k-1)(k-2) r_{1}^{k-2}}{2 n^{2}} \\
& \times\left[4 k\left(1+r_{1}\right)+(k-2)+r_{1}(k-1)\right] \\
& \leq r_{1}\left|E_{k-1, n}\left(z_{1}-i_{1} z_{2}\right)\right| \\
& +\frac{5\left(1+r_{1}\right)^{2} k(k-1)(k-2) r_{1}^{k-2}}{2 n^{2}} .
\end{aligned}
$$


With a similar way,

$$
\begin{aligned}
\left|E_{k, n}\left(z_{1}+i_{1} z_{2}\right)\right| & \leq r_{2}\left|E_{k-1, n}\left(z_{1}+i_{1} z_{2}\right)\right| \\
& +\frac{5\left(1+r_{2}\right)^{2} k(k-1)(k-2) r_{2}^{k-2}}{2 n^{2}}
\end{aligned}
$$

is found. Since $E_{0, n}\left(z_{1}+i_{2} z_{2}\right)=E_{1, n}\left(z_{1}+i_{2} z_{2}\right)=E_{2, n}\left(z_{1}+i_{2} z_{2}\right)=0$, for all $\left(z_{1}+i_{2} z_{2}\right) \in$ $\mathbb{C}_{2}$ and $k=0,1,2$, then we can write, for $k=3,4, \ldots$,

$$
\begin{aligned}
\left|E_{k, n}\left(z_{1}-i_{1} z_{2}\right)\right| & \leq \frac{5\left(1+r_{1}\right)^{2} r_{1}^{k-2}}{2 n^{2}} \sum_{j=3}^{k} j(j-1)(j-2) \\
& \leq \frac{5\left(1+r_{1}\right)^{2} r_{1}^{k-2}}{2 n^{2}} k(k-1)(k-2)^{2}
\end{aligned}
$$

and,

$$
\begin{aligned}
\left|E_{k, n}\left(z_{1}+i_{1} z_{2}\right)\right| & \leq \frac{5\left(1+r_{2}\right)^{2} r_{2}^{k-2}}{2 n^{2}} \sum_{j=3}^{k} j(j-1)(j-2) \\
& \leq \frac{5\left(1+r_{2}\right)^{2} r_{2}^{k-2}}{2 n^{2}} k(k-1)(k-2)^{2} .
\end{aligned}
$$

As a result, by using (A1) inequality:

$$
\begin{aligned}
& \left|\left(B_{n} f\right)\left(z_{1}-i_{1} z_{2}\right)-f\left(z_{1}-i_{1} z_{2}\right)-\frac{\left(z_{1}-i_{1} z_{2}\right)\left(1-\left(z_{1}-i_{1} z_{2}\right)\right)}{2 n} D_{z_{1}-i_{1} z_{2}}^{2}(f)\right| \\
& \leq \sum_{k=3}^{\infty}\left|c_{k}\right|\left|E_{k, n}\left(z_{1}-i_{1} z_{2}\right)\right| \\
& \leq \frac{5\left(1+r_{1}\right)^{2}}{2 n^{2}} M_{r_{1}}(f)
\end{aligned}
$$

and a similar way, from (A2), the following inequality:

$$
\begin{aligned}
& \left|\left(B_{n} f\right)\left(z_{1}+i_{1} z_{2}\right)-f\left(z_{1}+i_{1} z_{2}\right)-\frac{\left(z_{1}+i_{1} z_{2}\right)\left(1-\left(z_{1}+i_{1} z_{2}\right)\right)}{2 n} D_{z_{1}+i_{1} z_{2}}^{2}(f)\right| \\
& \leq \frac{5\left(1+r_{2}\right)^{2}}{2 n^{2}} M_{r_{2}}(f)
\end{aligned}
$$

is found. Furthermore, $M_{r_{1}}(f)$ and $M_{r_{2}}(f)$ are finite as in Theorem 3. Therefore, the theorem has been proved.

\section{Appendix C. Proof of Theorem 5}

For all $\left(z_{1}-i_{1} z_{2}\right) \in \mathbf{D}_{r_{1}}$ and $n \in \mathbb{N}$, we can write:

$$
\begin{aligned}
\left(B_{n} f\right)\left(z_{1}-i_{1} z_{2}\right)-f\left(z_{1}-i_{1} z_{2}\right) & =\frac{1}{n}\left\{\frac{\left(z_{1}-i_{1} z_{2}\right)\left(1-\left(z_{1}-i_{1} z_{2}\right)\right)}{2} D_{z_{1}-i_{1} z_{2}}^{2}(f)\right. \\
+\frac{1}{n}\left[n ^ { 2 } \left(\left(B_{n} f\right)\left(z_{1}-i_{1} z_{2}\right)-f\left(z_{1}-i_{1} z_{2}\right)\right.\right. & \left.\left.\left.-\frac{\left(z_{1}-i_{1} z_{2}\right)\left(1-\left(z_{1}-i_{1} z_{2}\right)\right)}{2 n} D_{z_{1}-i_{1} z_{2}}^{2}(f)\right)\right]\right\} .
\end{aligned}
$$

If $D_{z_{1}-i_{1} z_{2}}^{2}(f)$ is not equal to zero identically on $\mathbf{D}_{r_{1}}$ then there exists $M_{0}=\inf \left|z_{1}-i_{1} z_{2}\right|=r_{0}$ $\left|D_{z_{1}-i_{1} z_{2}}^{2}(f)\right|>0$ such that $0<r_{0}<1$. Now, let $r_{1}^{*} \geq 1$ be arbitrary. Clearly, we have 
$\left\|B_{n} f-f\right\|_{r_{1}^{*}} \geq\left\|B_{n} f-f\right\|_{r_{0}}$ there exists a point $\left(z_{0}-i_{1} z_{0}^{\prime}\right) \in \mathbb{C}$ depending on $f, n$ and $r_{0}$ with $\left|z_{0}-i_{1} z_{0}^{\prime}\right|=r_{0}$, such that:

$$
\begin{aligned}
\left\|B_{n} f-f\right\|_{r_{1}^{*}} & \geq\left\|B_{n} f-f\right\|_{r_{0}} \\
& \geq\left|\left(B_{n} f\right)\left(z_{0}-i_{1} z_{0}^{\prime}\right)-f\left(z_{0}-i_{1} z_{0}^{\prime}\right)\right| \\
& \geq \frac{1}{n} \mid\left\{\left|\frac{\left(z_{0}-i_{1} z_{0}^{\prime}\right)\left(1-\left(z_{0}-i_{1} z_{0}^{\prime}\right)\right)}{2} D_{z_{0}-i_{1} z_{0}^{\prime}}^{2}(f)\right|\right. \\
& -\frac{1}{n}\left[n ^ { 2 } \left(\mid\left(B_{n} f\right)\left(z_{0}-i_{1} z_{0}^{\prime}\right)-f\left(z_{0}-i_{1} z_{0}^{\prime}\right)\right.\right. \\
& \left.\left.\left.-\frac{\left(z_{0}-i_{1} z_{0}^{\prime}\right)\left(1-\left(z_{0}-i_{1} z_{0}^{\prime}\right)\right)}{2 n} D_{z_{0}-i_{1} z_{0}^{\prime}}^{2}(f) \mid\right)\right]\right\} \mid
\end{aligned}
$$

Now, we consider the first term and second term on the right side of the last inequality. We have:

$$
\left|\frac{\left(z_{0}-i_{1} z_{0}^{\prime}\right)\left(1-\left(z_{0}-i_{1} z_{0}^{\prime}\right)\right)}{2} D_{z_{0}-i_{1} z_{0}^{\prime}}^{2}(f)\right| \geq \frac{r_{0}\left(1-r_{0}\right)}{2} M_{0}>0
$$

and by Theorem 4:

$$
\begin{aligned}
& n^{2}\left|\left(B_{n} f\right)\left(z_{0}-i_{1} z_{0}^{\prime}\right)-f\left(z_{0}-i_{1} z_{0}^{\prime}\right)-\frac{\left(z_{0}-i_{1} z_{0}^{\prime}\right)\left(1-\left(z_{0}-i_{1} z_{0}^{\prime}\right)\right)}{2 n} D_{z_{0}-i_{1} z_{0}^{\prime}}^{2}(f)\right| \\
& \leq \frac{5 K_{r_{1}}(f)\left(1+r_{1}\right)^{2}}{2} .
\end{aligned}
$$

Therefore, for every $n \geq n_{0}$ there exists $n_{0}$ depending on $f$ and $r_{1}$ so that the following inequality is satisfied:

$$
\begin{aligned}
& \left|\frac{\left(z_{0}-i_{1} z_{0}^{\prime}\right)\left(1-\left(z_{0}-i_{1} z_{0}^{\prime}\right)\right)}{2 n} D_{z_{0}-i_{1} z_{0}^{\prime}}^{2}(f)\right|-\frac{1}{n}\left[n^{2} \mid\left(B_{n} f\right)\left(z_{0}-i_{1} z_{0}^{\prime}\right)\right. \\
& \left.-f\left(z_{0}-i_{1} z_{0}^{\prime}\right)-\frac{\left(z_{0}-i_{1} z_{0}^{\prime}\right)\left(1-\left(z_{0}-i_{1} z_{0}^{\prime}\right)\right)}{2 n} D_{z_{0}-i_{1} z_{0}^{\prime}}^{2}(f) \mid\right] \\
& \geq \frac{r_{0}\left(1-r_{0}\right)}{4} M_{0} .
\end{aligned}
$$

which implies $\left\|B_{n} f-f\right\|_{r_{1}^{*}} \geq \frac{r_{0}\left(1-r_{0}\right)}{4} M_{0}$, for all $n \geq n_{0}$. For $n \in\left\{1,2, \ldots, n_{0}-1\right\}$, one obtain $\left\|B_{n} f-f\right\|_{r_{1}^{*}} \geq \frac{M_{r_{1, n}^{*}}(f)}{n}$ and $M_{r_{1, n}^{*}}(f)=n\left\|B_{n} f-f\right\|_{r_{1}^{*}}>0$. Therefore, for every $n \in \mathbb{N},\left\|B_{n} f-f\right\|_{r_{1}^{*}} \geq \frac{C_{r_{1}^{*}}(f)}{n}$ is satisfied, where:

$$
C_{r_{1}^{*}}(f)=\min \left\{M_{r_{1,1}^{*}}(f), M_{r_{1,2}^{*}}(f), \ldots, M_{r_{1, n_{0}-1}^{*}}(f), \frac{r_{0}\left(1-r_{0}\right)}{4} M_{0}\right\}
$$

The other case can be shown in a similar manner. 


\section{References}

1. Soleymani, F.; Stanimirović, P.S.; Torregrosa, J.R.; Nik, H.S.; Tohidi, E. Recent Theories and Applications in Approximation Theory. Sci. World J. 2015, 2015, 598279. [CrossRef] [PubMed]

2. Korovkin P.P. Linear Operators and Approximation Theory; Hindustan Publishing Corporation: Delhi, India, 1960.

3. Lorentz, G.G. Bernstein Polynomials, 2nd ed.; Chelsea Publ.: New York, NY, USA, 1986.

4. Voronovskaja, E.V. Determination de la forme asymptotique de I' approximation des fonctions par les polynomes de M. Bernstein. C. R. Acad. Sci. URSS 1932, 1, 79-85.

5. Ciupa, A. A Voronovskaya-type theorem for a positive linear operators. Int. J. Math. Math. Sci. 2006, 1-7. [CrossRef]

6. Rempulska, I.; Skorupka, M. The Voronovskaya Theorem for some operators of Szasz-Mirakyan Type. Le Matematiche L 1995, 9 , 251-261.

7. Bardaro, C.; Mantellini, I. A Voronovskaja-type theorem for a general class of discrete operators. Rocky Mt. J. Math. 2009, 39, 1411-1442. [CrossRef]

8. Tonne, P.C. On the Convergence of Bernstein Polynomials for Some Unbounded Analytic Functions. Proc. Am. Math. Soc. 1969, 22, 1-6. [CrossRef]

9. Gal, S.G. Voronovskaja's theorem and iterations for complex Bernstein polynomials in compact disks. Mediterr. J. Math. 2008, 5, 253-272. [CrossRef]

10. Gal, S.G. Shape Preserving Approximation by Real and Complex Polynomials; Birkhauser Publ.: Boston, FL, USA, 2008.

11. Gal, S.G. Approximation by Complex Bernstein and Convolution Type Operators; Series on Concrete and Applicable Mathematics; World Scientific: Singapore, 2009; Volume 8, p. 352.

12. Mursaleen, M.; Nasiruzzaman, M.; Srivastava, H.M. Approximation by bicomplex beta operators in compact BC-disks. Math Methods Appl. Sci. 2016, 39, 2916-2929. [CrossRef]

13. Pogorui, A.A.; Rodríguez-Dagnino, R.M. On the set of zeros of bicomplex polynomials. Complex Var. Elliptic Equ. 2006, 51, 725-730. [CrossRef]

14. Wagh, M.A. Some Studies and Applications of Bicomplex Numbers. In Proceedings of the International Workshop on Emerging Opportunities and Trends in Basic and Applied Sciences, Dbrau, India, 22-24 January 2016. [CrossRef]

15. Segre, C. The real representation of complex elements and hyperalgebraic entities (Italian). Math. Ann. 1892, 40, $413-467$. [CrossRef]

16. Rochon, D.; Shapiro, M. On algebraic properties of bicomplex and hyperbolic numbers. Anal. Univ. Oradea Fasc. Math. 2004, 11, 71-110.

17. Price, G.B. An Introduction to Multicomplex Spaces and Functions; Monographs and Textbooks in Pure and Applied Mathematics; M. Dekker: New York, NY, USA, 1991; Volume 140.

18. Rönn, S. Bicomplex algebra and function theory. arXiv 2001, arXiv:math/0101200v1.

19. Arfaoui, S.; Mabrouk, A.B.; Cattani, C. New type of Gegenbauer-Jacobi-Hermite monogenic polynomials and associated continuous Clifford wavelet transform. Acta Appl. Math. 2020, 1-35. [CrossRef]

20. Arfaoui, S.; Mabrouk, A.B.; Cattani, C. Wavelet Analysis: Basic Concepts and Applications; CRC Press: Boca Raton, FL, USA; London, UK; New York, NY, USA, 2021.

21. Jallouli, M.; Arfaoui, S.; Mabrouk, A.B.; Cattani, C. Clifford Wavelet for fetal ECG extraction. arXiv 2021, arXiv: 2103.04085.

22. Gargoubi, H.; Kossentini, S. Bicomplex Numbers as a Normal Complexified f-Algebra. Hal-02875956. 2020. Available online: https: / / scholar.google.com/scholar?q=Gargoubi\%2C\%20H.\%3B\%20Kossentini\%2C\%20S.\%20Bicomplex\%20numbers\% 20as $\% 20 \mathrm{a} \% 20$ normal $\% 20$ complexified $\% 20 \mathrm{f} \% 20$-algebra (accessed on 28 October 2021).

23. Usman, T.; Saif, M.; Choi, J. Certain identities associated with (p;q)-binomial coefficients and (p;q)-Stirling polynomials of the second kind. Symmetry 2020, 12, 1436. [CrossRef]

24. Futagawa, M. On the theory of functions of quaternary variable-I. Tohoku Math. J. 1928, 29, 175-222.

25. Futagawa, M. On the theory of functions of quaternary variable-II. Tohoku Math. J. 1932, 35, 69-120.

26. Sabadini, I.; Vajiac, A.; Vajiac, M.B. Bernstein-type Inequalities for Bicomplex Polynomials. In Advances in Complex Analysis and Operator Theory; Colombo, F., Sabadini, I., Struppa, D., Vajiac, M., Eds.; Trends in Mathematics; Birkhäuser: Cham, Switzerland, 2017.

27. Luna-Elizarrarás, M.E.; Shapiro, M.; Struppa, D.C.; Vajiac, A. Bicomplex Holomorphic Functions: The Algebra, Geometry and Analysis of Bicomplex Numbers; Birkhauser: Cham, Switzerland, 2015.

28. Luna-Elizarrarás, M.E.; Panza, M.; Shapiro, M. Geometry and Identity Theorems for Bicomplex Functions and Functions of a Hyperbolic Variable. Milan J. Math. 2020, 88, 247-261. [CrossRef]

29. Luna-Elizarrarás, M.E.; Shapiro, M.; Struppa, D.C. Bicomplex numbers and their elementary functions. Cubo 2012, 14, 61-80. [CrossRef]

30. Datta, S.K.; Molla, T.; Sk, M.; Saha, J. A note on the bicomplex version of Enström-Kakeya Theorem. J. Xi'An Univ. Archit. Technol. 2020, 12, 593-603.

31. Rochon, D.; Tremblay, S. Bicomplex Quantum Mechanics: II. the Hilbert space. Adv. Appl. Clifford Algebr. 2006, 16, 135-157. [CrossRef]

32. Gürlebeck, K.; Sprössig, W. Quaternionic and Clifford Calculus for Physicists and Engineers; John Wiley and Sons: Hoboken, NJ, USA, 1997. 
33. Kravchenko, V.; Shapiro, M. Integral Representations for Spatial Models of Mathematical Physics; Pitman Research Notes in Math; Addison-Wesley-Longman: Boston MA, USA 1996; Volume 351, p. 247.

34. Goswami, M.P.; Jha, N. Triple Laplace Transform in Bicomplex Space with Application. Math. Stat. 2020, 8, 443-450. [CrossRef]

35. Kobayashi, M. Stability Conditions of Bicomplex-Valued Hopfield Neural Networks. Neural Comput. 2021, 33, 552-562. [CrossRef]

36. Kobayashi, M. Bicomplex Projection Rule for Complex-Valued Hopfield Neural Networks. Neural Comput. 2020, 32, $2237-2248$. [CrossRef] 